\title{
Parametric Study on Performance of Inertance Pulse Tube Cryocooler
}

\author{
K.H. Lee*
}

Mechanical Engineering Department, Sunchon National University, Sunchon, Republic of Korea

\section{J. Rhee**}

Korea Aerospace Research Institute, Daejeon, Republic of Korea

\author{
J.S. Kim*** \\ Department of Aerospace Engineering, Chosun University, Gwangju, Republic of Korea
}

\begin{abstract}
The design parameters to affect the cooling capacity of a cryocooler were examined with the application of numerical modeling to optimize an inertance pulse tube cryocooler. This modeling includes the regenerator, pulse tube, inertance tube, gas reservoir, and heat exchangers. One-dimensional modeling on strings of acoustic and thermoacoustic elements was applied to compare the design parameters. The diameter and length of the pulse tube can significantly affect the cooling capacity and efficiency. The aftercooler was optimized by maintaining a certain size. The efficiency also improved as the length of inertance tube and volume of gas reservoir are increased. It was confirmed that effective design parameters are critical to the performance of an inertance pulse tube cryocooler considering the comparison of the dimensions of each part to optimize its cooling power and efficiency.
\end{abstract}

Key words: Inertance tube, Pulse tube cryocooler, Wave equation, Regenerator

\section{Introduction}

The cryocooler has been widely applied in spacecraft missions, for military purposes, and in medical equipment. The need for high sensitivity in an infrared imaging instrument requires cryogenic cooling in order to lower the background photon noise levels and to lower any residual dark current or thermally induced noise in the detector itself. Similarly, cryocoolers are necessary to reduce detector noise in gamma-ray spectrometers and shot noise in detector amplifier systems. Superconducting technologies and medical instruments inherently need cryogenic cooling of RF filters for super high conductivity [1].

Especially for space applications, coolers need to tolerate long term use of more than 5 years with enough reliability, in high acceleration due to gravity, electromagnetic barriers, and space radiation. Although there are various cryocoolers, the pulse tube cryocooler is operated by a gas piston that doesn't need a mechanical driving system and that repeats the compression and expansion of refrigerant. These methods not only reduce the vibration and noise but also improve the reliability and durability [2].

Since the inertance pulse tube cryocooler doesn't have any mechanical mechanism, it has a relatively simple structure which doesn't need a complicated manufacturing process or assembling technology. Compared to Stirling cryocoolers and GM cryocoolers, it has many advantages such as low manufacture price and high reliability. In terms of durability, pulse tube cryocooler has at least 5 year lifespan, while some systems currently have been operating for more than 10 years [3]. It can be flexibly applied in various cooling conditions, with relatively low pressure ratio and charging pressure. However,
This is an Open Access article distributed under the terms of the Creative Commons Attribution Non-Commercial License (http://creativecommons.org/licenses/by$\mathrm{nc} / 3.0 /$ ) which permits unrestricted non-commercial use, distribution, and reproduction in any medium, provided the original work is properly cited. (c) * Professor
$* *$ Corresponding Author : jrhee@kari.re.kr
*** Professor 
the basic pulse tube cryocooler has low heat efficiency and low cooling ability, as well as low practicality because of high cooling temperature. In order to better control the pressure and mass flow within the system, the orifice pulse tube cryocooler was developed. The orifice is installed between the pulse tube and the gas reservoir to control the phase between mass flow and the pressure wave. However, this type of pulse tube cryocooler has some problems due to the the operation of orifice.

The pulse tube cryocooler with an inertance tube is one of the most effective cryocoolers for space applications, especially for cooling infrared detectors [4]. It has many advantages because of its reliability (due to lack of moving parts at the cold end), simplicity in structure, low vibration, long operating life, and relatively high efficiency. It consists of a compressor with a linear motor, a regenerator, a pulse tube, an inertance tube, a reservoir, and heat exchangers. The regenerator with porosity is the main component that improves the cooling power with the regeneration of heat flow. The pulse tube is applied to expand the compressed operating gas without any moving parts in contrast to a gas expander with a piston. This type of cryocooler is characterized by the use of a long and thin tube for phase shifting which improves the performance at the hot end of pulse tube. Since the phase angle between the pressure wave and mass flow is influenced by the wave resonance phenomena, the optimal phase lag in the pulse tube is the most critical factor to affect the performance of this type of cryocooler [5]. The role of phase shifting is done by the long tube between the buffer volume and the high temperature part of the pulse tube.

This study investigated the design parameters that affect the cooling capacity of the cryocooler, in particular the dimensions of the regenerator, the pulse tube, the inertance tube, the gas reservoir, and the heat exchangers by optimizing the system through numerical modeling. Through comparing and reviewing the changes in the numerical analysis of the cryocooler, the most essential and economic factors for actually designing the pulse tube cryocooler were examined that would be used in space application.

\section{Inertance pulse tube cryocooler}

\subsection{Structure and operating characteristics}

The basic structure of the inertance tube pulse tube cryocooler is shown in Fig. 1. It contains a compressor, a regenerator, a pulse tube, an inertance tube, a reservoir, and heat exchangers (aftercooler (AC), cold heat exchanger
(CHX), and hot heat exchanger (HHX)).

From the inertance tube pulse tube cryocooler, the gas within inertance tube vibrates with the expansion work done by the gas in the pulse tube. At this time, the standing wave and progressive wave occurs in the inertance tube. The standing wave changes from a mass wave to a pressure wave, and a progressive wave changes the expansion work to heat. The pressure amplitude in the regenerator slightly decreases due to the pressure loss. The work flow within the pulse tube occurs as the pressure amplitude of the inertance tube decreases. The energy flow from the compressor outlet to the hot side of the pulse tube is similar with to basic pulse tube cryocooler. The work flow in the inertance tube decreases to 0 at the reservoir connection. The enthalpy flow is a little lower than the flow of the work inside inertance tube, and decreases to 0 at the reservoir connection. The heat flow near the hot side of pulse tube is almost 0 and it decreases to a negative (-) value along the inertance tube and becomes 0 at

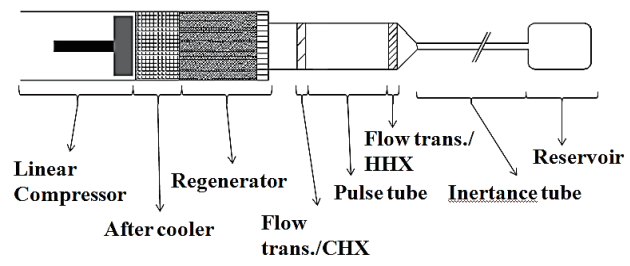

Fig. 1. Schematic diagram of inertance pulse tube cryocooler [5]
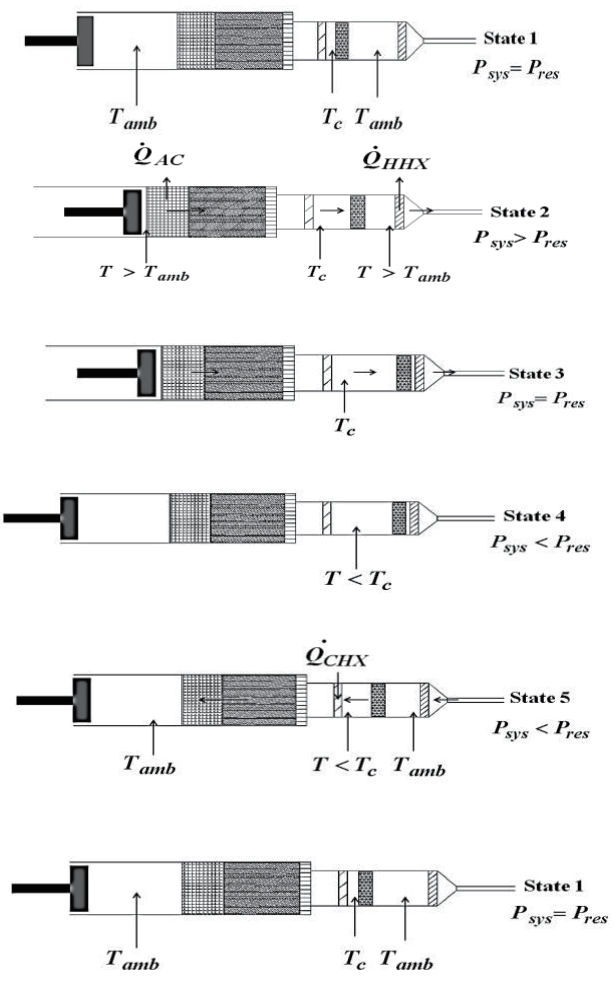

Fig. 2. Operating process of inertance pulse tube cryocooler 
the reservoir connection.

The temperature of the cold end keeps decreasing as the cycle repeats compared to preceding cycle. The final lowest temperature is reached at the equilibrated state between the cooling load and the cooling capacity. The pulse tube which is operated in this process, it shows insufficient performance and efficiency compared to other coolers. But, the structures are simple, and its reliability and durability are very outstanding. Since its production is simple and the price is inexpensive, there are many advantage points over other existing cryogenic freezers and it has replaced them.

The ideal cycle of the pulse tube cryocooler consists of 5 states that can be seen in Fig. 2, the operating process is as follows:

(1-2) When the piston of the compressor is compressed, the gas goes toward the reservoir which is at the opposite end of the system. As the gas temperature rises, it dissipates heat from the aftercooler to the surroundings. Then, it passes the regenerator and dissipates heat to the filler so it precools before entering the pulse tube's cold end. The temperature of the gas increases as the pressure of the gas from the pulse tube increases. The gas piston developed from the gas movement within the pulse tube starts to move towards the reservoir volume along the pulse tube. As this gas passes the heat exchanger into the reservoir, additional heat is released.

(2-3) During the course of the compression cycle, the gas flow continues until the combination of gas entering the reservoir coupled with the heat release to the surroundings in the hot heat exchanger is sufficient to cause the system pressure to equilibrate with the mean pressure in the reservoir.

(3-4) As the piston of the compressor expands to the left, the gas rapidly expands and the temperature drops lower than the heat generator.

(4-5) Through the process of expanding, the pressure of the system decreases more than the reservoir pressure and the gas flows to the compressor end of the system. As the gas flows towards the compressor, cold gas inside the pulse tube flows through the low temperature heat exchanger to absorb heat to cool the object

As this gas passes through the regenerator, the temperature of the gas increases with the accumulated heat from the previous cycle.

(5-1) From the last stage of cycle, the pressure within the system again balances with the reservoir pressure and stops the flow of gas. At this time, the cycle is repeated.

In Fig. 2, $T_{a m b}, T_{c}, P_{s y s}, P_{r e s}$, and $\dot{Q}$ are the ambient temperature, temperature in cold heat exchanger, pressure of system, pressure in reservoir, and the heat flux, respectively.

\subsection{Design of inertance pulse tube cryocooler}

\subsubsection{Type decisions and design considerations}

When designing a cryocooler, the factors to be considered are cooling temperature, refrigeration load, reliability, vibration, temperature controllability, cooling time, input power, volume, weight, installation environment, cost, efficiency, and materials. The specific design parameters are the compression ratio, the volume ratio, operating pressure, the phase angle of compression and expansion, pore volume, speed of operation, inflator, the diameter and stroke of the compressor, the arrangement of the magnet and coil, spring constant, separation tube, material, the type of working fluid, driver, the regenerator material and structure, the configuration of the heat exchanger, and sealing of the device. There are also differences between an actual system and theoretical designs. The pressure drop at the heat regenerator, the temperature difference between the gas and the wall at the heat exchanger, the temperature difference between regenerator material and the gas, hysteresis losses due to heat transfer from the compressor and expander, heat transfer between the expander and piston, conduction and convection heat transfer between high and low temperatures, and the loss by the gap between piston and cylinder all need to be reviewed [6].

In terms of reliability, the factors to consider are the concentricity to minimize the friction between the cylinder and the piston, sealing and wear resistance of the piston ring, minimization of spring deformation, working gas leak in the cooler, and dust removal. Additionally, a linear motor where operating frequency and driving force are adjustable and having a low sidewall force should be suitable. In order to minimize the contact of the support structure and the bearing, a flexure bearing should be used. To set the operating conditions, determination of the structure's caloric value, output and input power, and refrigerant should be considered.

\subsubsection{Application of design program}

The entire system has to be created by combining various dimensions of each part shown in Fig. 1 through many trials to analyze the final cooling performance for the determination of the specifications of the cryocooler. The design program used in this study was DELTA-EC (Design Environment for Low-amplitude Thermoacoustic Energy Conversion) developed in Los Alamos. Basically, the numerical modeling of the motion of the operating gas in the system adopted the application of a one dimensional second order wave equation without viscous or thermal-hysteresis losses [7]. It is regarded as two coupled first order equations 
in pressure and volume flow rate which are derived from the momentum equation and continuity equation that includes dissipation of acoustic power along the sides of ducts. This wave equation expresses pressure amplitude $p_{1}$ by using second order of the Helmholtz differential equation as

$$
p_{1}+\frac{a^{2} d^{2} p_{1}}{\omega^{2} d x^{2}}=0
$$

where $a$ is sound speed and $\omega$ is radian frequency.

The linear thermoacoustic theory for the thermoacoustic effect was established by Rott [8] and summarized later by Swift $[9,10]$. The approximations used by the linear thermoacoustic theory are as follows:

$$
\begin{aligned}
& p=p_{m}+\operatorname{Re}\left[p_{1}(x) e^{i \omega x}\right], \\
& U=\operatorname{Re}\left[U_{1}(x) e^{i \omega x}\right], \\
& T=T_{m}+\operatorname{Re}\left[\rho_{1}(x) e^{i \omega x}\right], \\
& \rho=\rho_{m}+\operatorname{Re}\left[\rho_{1}(x) e^{i \omega x}\right] .
\end{aligned}
$$

From the above equations, equation (6) was derived to quantitatively describe the interaction between the acoustic and temperature fields.

$$
\begin{gathered}
{\left[1+(\gamma-1) f_{k}\right] p_{1}+\frac{\gamma p_{1}}{\omega^{2}} \frac{d}{d x}\left(\frac{1-f_{v}}{\rho_{m}}\right)} \\
-\frac{a^{2}}{\omega^{w}} \frac{f_{k}-f_{v}}{1-\sigma} \frac{1}{T_{m}} \frac{d T_{m}}{d x} \frac{d p_{1}}{d x}=0
\end{gathered}
$$

Through the above equations, $p, U, T, \rho$, and $\gamma$ are the pressure, volumetric velocity, temperature, density and the ratio of specific heat capacities of the gas, respectively; $\omega$ and $a$ are the angular frequency and speed of the acoustic wave; $f_{k}$ and $f_{v}$ which depend on the size and shapes of the pores and on the gas properties are the spatially averaged thermal and viscous functions, respectively. Subscript ' 1 ' indicates the first order of a variable, which usually has a complex amplitude. For the convenience of numerical calculations,

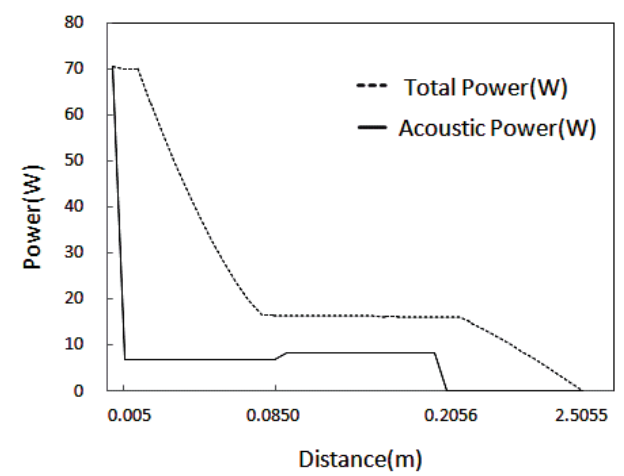

Fig. 3. Typical example of trace of total power and acoustic Power in the system equation (6) can be written as:

$$
\begin{aligned}
d p_{1}= & -\frac{\frac{i \omega \rho_{m} d x}{A}}{1-f_{v}} U_{1}, \\
d U_{1}= & -\frac{i \omega \rho_{m} A d x}{\gamma p_{m}}\left[1+(\gamma-1) f_{k}\right] p_{1} \\
& +\frac{\left(f_{k}-f_{v}\right)}{\left(1-f_{v}\right)(1-\sigma)} \frac{d T_{m}}{T_{m}} U_{1}
\end{aligned}
$$

In essence, equations (7) and (8) are the thermoacoustic version of momentum and continuity equations. Two more equations for the temperature $T_{m}(\mathrm{x})$ and total power $\dot{H}_{t o t}(\mathrm{x})$ are required to complete this calculation. These equations depend on the type of segment and continuity of $T_{m}$ and $\dot{H}_{t o t}$ which are imposed at the junctions between segments. Most segments simply obey $d T_{m}(\mathrm{x}) / d x=0$ and $d \dot{H}_{t o t}(\mathrm{x}) / d \mathrm{x}=0$. Some segments, though, have a more complicated differential equation for $T_{m}(\mathrm{x})$, which is derived from Rott's energy equation [8]. The total power $\dot{H}_{t o t}$ changes with $\mathrm{x}$ in heat exchanger segments, according to

$$
\frac{d \dot{H}_{\text {tot }}}{d \mathrm{x}}=\dot{q}
$$

where $\dot{q}$ is the heat added per unit length in the heat exchanger.

A shooting method is used by guessing any unknowns among the numbers defining pressure and volume flow rate at the initial end of the integration comparing the results with the target boundary conditions imposed at the other end or elsewhere, and adjusting its guessed value until the integration results meet the targets. The calculation process using first order ordinary differential equations is performed by the numerical iteration method. However, it is not just getting a solution by simple initial condition; it starts the calculation with the outlet of compressor as the inlet condition. The problem should be satisfied with the required conditions at each step in the system. The requirements at each step are 4 conditions, which are the wall temperature in the cold exchanger and hot exchanger and the adiabatic

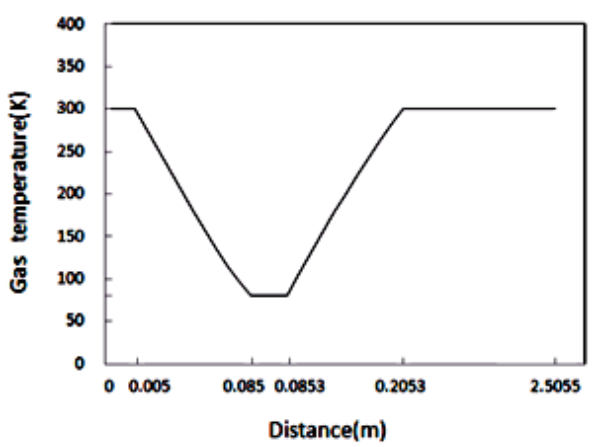

Fig. 4. Typical example of trace of temperature according to the location within system 
and blocked wall condition. Since the ordinary differential equations have to be calculated from the initial value, it should be repeatedly calculated with 4 assumed values to satisfy the target required at each step.

A typical example of the trace of change in total power and acoustic power within the system by DELTA-EC is shown in Fig. 3. The total power decreases from the compressor to the regenerator. And then, it starts to decrease again from the inertia tube after it maintains the total power constantly in the pulse tube. Acoustic power decreases sharply at the end of the pulse tube.

The change in temperature within the system is also shown in Fig. 4. In this case, when the initial temperature is $300 \mathrm{~K}$, the temperature reduces from the heat regenerator and the coolers $80 \mathrm{~K}$. Then the temperature at the pulse tube entrance maintains this temperature and the temperature begins to increase at the hot heat exchanger.

\section{Results}

\subsection{Effect of pulse tube, inertance tube, and reser- voir}

The effect of a pulse tube on cooling capacity and

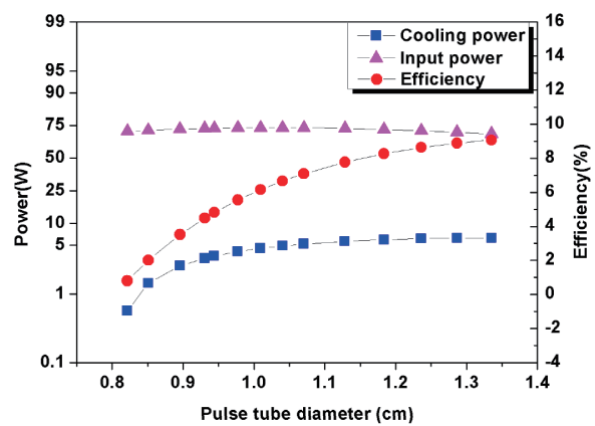

Fig. 5. Effect of pulse tube diameter on cooling power, input power, and efficiency

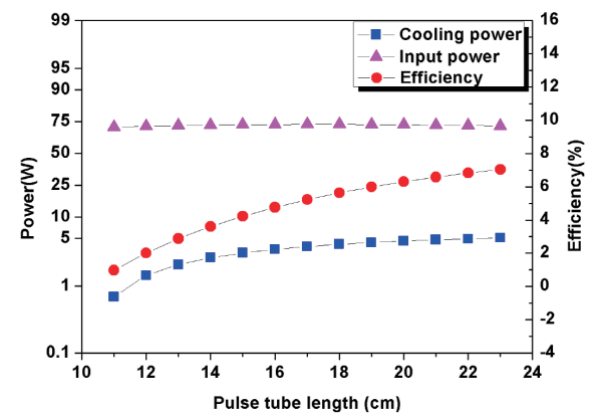

Fig. 6. Effect of pulse tube length on cooling power, input power, and efficiency efficiency was compared as the diameter and length of pulse tube changed. The effect of pulse tube diameter (Fig. 5) is that the cooling power increases rapidly as the diameter increases between 0.8 and $0.95 \mathrm{~cm}$, however, the effect diminishes as the diameter increases more than $1 \mathrm{~cm}$. The efficiency shows a little different trend; it continues to increase as the pulse diameter increases, due to the decrease in the input power. The effect of pulse tube length shows almost the same pattern as pulse tube diameter (Fig. 6 ). The cooling power increases relatively rapidly to $14 \mathrm{~cm}$, but then the effect doesn't seem to be significant. The efficiency continues increasing as the case of pulse tube diameter.

The length of inertance tube shows almost a linear increase as the length increases (Fig. 7). Even the input doesn't seem to change drmatically, the cooling power and efficiency increase as the length increases. However, there should be a restriction on the length of inertance tube due to the limit on the size of cryocooler. Increasing inertance length has less of an effect on cooling power than changes in the pulse tube. The effect of reservoir volume on cooling power, input power, and efficiency is shown in Fig. 8. As the reservoir volume increases, the cooling power and efficiency increase. However, it doesn't seem as significant as other parameters.

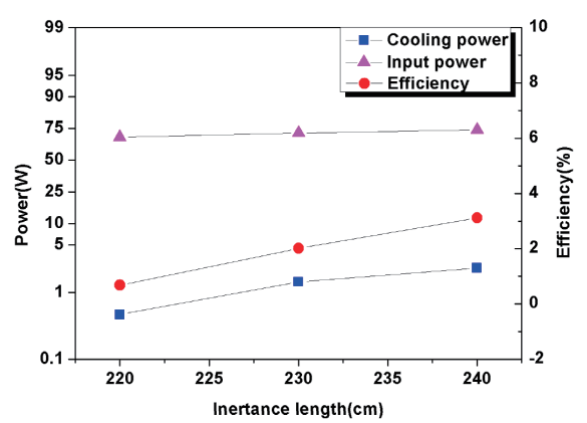

Fig. 7. Effect of inertance length on cooling power, input power, and efficiency

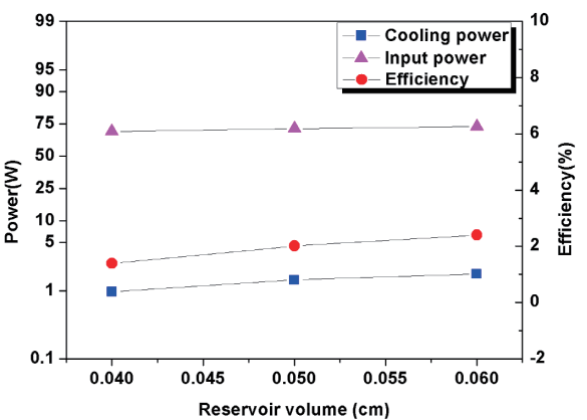

Fig. 8. Effect of reservoir volume on cooling power, input power, and efficiency 


\subsection{Effect of regenerator, aftercooler, and heat ex- changer}

The result of changes in the length of the regenerator shows a different trend (Fig. 9). The cooling capacity and efficiency doesn't increase over $10 \mathrm{~cm}$ and seems to have the optimum performance at around $10 \mathrm{~cm}$ in length. The input power decreases slightly as the regenerator length increases.

The effect of changes in the length of cold heat exchanger is shown in Fig. 10. As the length of the cold heat exchanger increases, the cooling power and efficiency decrease, however, input power stays constant. As the diameter of the aftercooler increases, the cooling power and efficiency

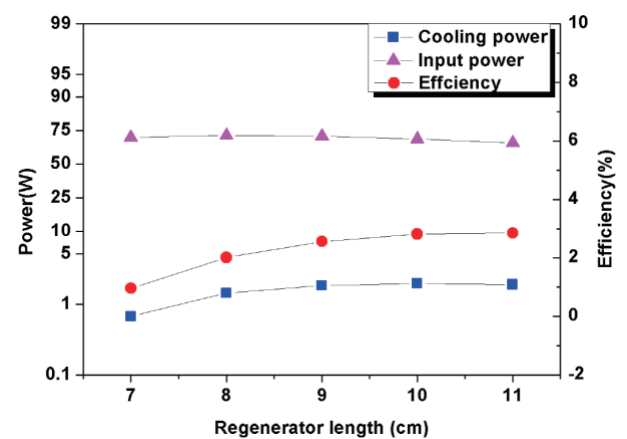

Fig. 9. Effect of regenerator length on cooling power, input power, and efficiency

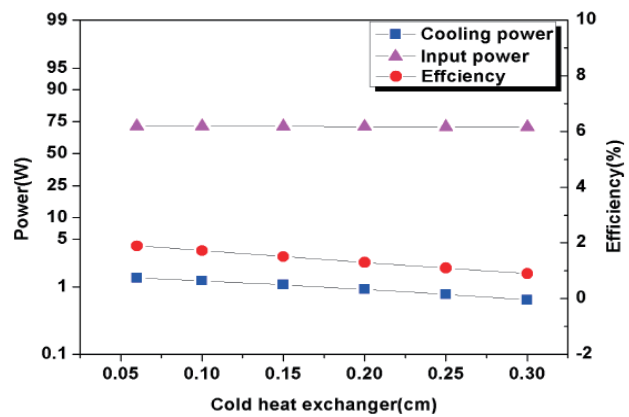

Fig. 10. Effect of length of cold heat exchanger on cooling power, input power, and efficiency

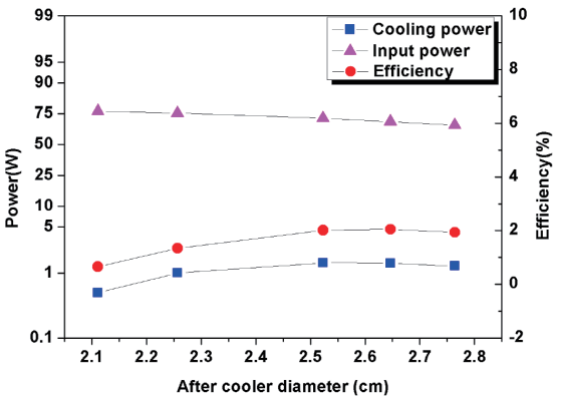

Fig. 11. Effect of aftercooler diameter on cooling power, input power, and efficiency increases until it reaches $2.5 \mathrm{~cm}$ when they remain constant or drop (Fig. 11). The optimum value of the aftercooler diameter seems to be $2.5 \mathrm{~cm}$.

\section{Conclusions}

Through the numerical analysis on the inertance pulse tube cryocooler, the design parameters which can affect the cooling capacity and efficiency of a cryocooler were investigated and optimized. Through the comparison on the changes in the cryocooler specification, it is concluded as follows;

(1) The diameter and length of pulse tube can significantly affect the cooling capacity and efficiency. The cooling power doesn't increase significantly over $1.0 \mathrm{~cm}$ in diameter and $14 \mathrm{~cm}$ in length even though the efficiency keeps increasing.

(2) As the inertance length and reservoir volume increase the cooling performance also increases. However, the effect is not as significant as the dimensions of pulse tube.

(3) There seems to be an optimized size in the length of regenerator since these are limited at around $10 \mathrm{~cm}$ even though the length increases.

(4) The aftercooler diameter also shows an optimized size at $2.5 \mathrm{~cm}$ since the cooling power and efficiency increases to a certain level. Yet, they are not increased anymore but dropped as the size increases.

\section{Acknowledgment}

This study was supported by Korea Aerospace Research Institute.

\section{References}

[1] Bhatia, R., "Review of Spacecraft Cryogenic Coolers", Journal of Spacecraft and Rockets, Vol. 39, No. 3, 2002, pp. 329-346.

[2] Radebaugh, R., "Development of the Pulse Tube Refrigerator as an Efficient and Reliable Cryocooler ", Proc. Institute of Refrigeration, London 1999-2000.

[3] Radebaugh, R., "Pulse Tube Cryocoolers for Cooling Infrared sensors", Proceedings of SPIE, The International Society for Optical Engineering, Infrared Technology and Applications XXVI, Vol. 4130, 2000, pp. 363-379.

[4] Ross, R., and Boyle, R., "An Overview of NASA Space 
Cryocooler Program-2006", International Cryocooler Conference, Annapolis, MD, June 14-6, 2006.

[5] Kim, Y., and Chang, H., "Cryogenic Refrigerators", Journal of Air-Conditioning and Refrigeration, Vol. 19, No. 1, 1990, pp. 7-18.

[6] Taylor, R., "Optimal Pulse-Tube design Using Computational Fluid Dynamics", Ph.D Thesis, University of Wisconsin-Madison, 2009.

[7] Ward, B., Clark, J., and Swift, G., "Design Environment for Low-amplitude Thermoacoustic Energy Conversion DELTAEC Ver.6.2 Users Guide”, Los Alamos National Lab.,
2008. pp. 7-18.

[8] Rott, N., "Thermally driven acoustic oscillations, part III: Second-order heat flux", Z. Angew. Math Phys., Vol. 26, 1975, pp.43-49

[9] Swift, G.W., "Thermoacoustics: A Unifying Perspective for some Engines and Refrigerators", Acoustical Society of America, Publications, Sewickley PA., 2002.

[10] Abduljalil, A.S., Yu, Z., and Jaworski, A.J., "Design and Experimental Validation of Looped-tube Thermoacoustic Engine", J. of Thermal Science, vol.20, No. 5, 2011, pp. 423429 